\title{
Validity and responsiveness of EQ-5D-5L and SF-6D in patients with health complaints attributed to their amalgam fillings: a prospective cohort study of patients undergoing amalgam removal
}

\author{
Admassu N. Lamu ${ }^{1 *} \mathbb{D}$, Lars Björkman²,3 ${ }^{2}$ Harald J. Hamre ${ }^{4}$, Terje Alræk ${ }^{5}$, Frauke Musial ${ }^{5}$ and Bjarne Robberstad ${ }^{1}$
}

\begin{abstract}
Background: Evidence of health utility changes in patients who suffer from longstanding health complaints attributed to dental amalgam fillings are limited. The change in health utility outcomes enables calculating quality-adjusted life-year (QALY) and facilitates the comparison with other health conditions. The purpose of this study was to estimate the validity and responsiveness of the EQ-5D-5L and SF-6D utilities following removal of dental amalgam fillings in patients with health complaints attributed to their amalgam fillings, and examine the ability of these instruments to detect minimally important changes over time.

Methods: Patients with medically unexplained physical symptoms, which they attributed to dental amalgam restorations, were recruited to a prospective cohort study in Norway. Two health state utility instruments, EQ-5D-5L and SF-6D, as well as self-reported general health complaints (GHC-index) and visual analogue scale (EQ-VAS) were administered to all patients $(n=32)$ at baseline and at follow-up. The last two were used as criteria measures. Concurrent and predictive validities were examined using correlation coefficients. Responsiveness was assessed by the effect size (ES), standardized response mean (SRM), and relative efficiency. Minimally important change (MIC) was examined by distribution and anchor-based approaches.
\end{abstract}

Results: Concurrent validity of the EQ-5D-5L was similar to that of SF-6D utility. EQ-5D-5L was more responsive than SF-6D: the ES were 0.73 and 0.58 for EQ-5D-5L and SF-6D, respectively; SRM were 0.76 and 0.67 , respectively. EQ5D-5L was more efficient than SF-6D in detecting changes, but both were less efficient compared to criteria-based measures. The estimated MIC of EQ-5D-5L value set was 0.108 and 0.118 based on distribution and anchor-based approaches, respectively. The corresponding values for SF-6D were 0.048 and 0.064 , respectively.

Conclusions: In patients with health complaints attributed to dental amalgam undergoing amalgam removal, both EQ-5D-5L and SF-6D showed reasonable concurrent and predictive validity and acceptable responsiveness. The EQ5D-5L utility appears to be more responsive compared to SF-6D.

\footnotetext{
*Correspondence: admassu.lamu@uib.no; kanaroba2.12@gmail.com

1 Section for Ethics and Health Economics, Department of Global Public

Health and Primary Care, Faculty of Health Sciences, University of Bergen,

5020 Bergen, Norway

Full list of author information is available at the end of the article
}

(c) The Author(s) 2021. Open Access This article is licensed under a Creative Commons Attribution 4.0 International License, which permits use, sharing, adaptation, distribution and reproduction in any medium or format, as long as you give appropriate credit to the original author(s) and the source, provide a link to the Creative Commons licence, and indicate if changes were made. The images or other third party material in this article are included in the article's Creative Commons licence, unless indicated otherwise in a credit line to the material. If material is not included in the article's Creative Commons licence and your intended use is not permitted by statutory regulation or exceeds the permitted use, you will need to obtain permission directly from the copyright holder. To view a copy of this licence, visit http://creativecommons.org/licenses/by/4.0/. The Creative Commons Public Domain Dedication waiver (http://creativeco mmons.org/publicdomain/zero/1.0/) applies to the data made available in this article, unless otherwise stated in a credit line to the data. 
Trial registration The research was registered at ClinicalTrials.gov., NCT01682278. Registered 10 September 2012, https://clinicaltrials.gov/ct2/show/NCT01682278.

Keywords: EQ-5D-5L, SF-6D, Utility, Responsiveness, Validity, Minimally important change

\section{Background}

Dental amalgam is one of the most widely used dental restorative materials, but safety concerns relating to its mercury content have been a topic of discussion for many years [1]. This has been a particularly contentious issue in Norway, where amalgam use was banned in 2008, due to environmental considerations. Some people with amalgam fillings report health complaints with a pattern similar to patients with medically unexplained physical symptoms (MUPS), where the symptoms are non-specific and a known pathophysiology is missing [2, 3]. Furthermore, they attribute their health complaints to mercury released from their amalgam fillings [3]. Hereafter we refer to these patients as amalgam patients. Thus, assessing health outcomes in amalgam patients and their health-related quality of life is essential for clinical practice and future research strategies. Evidence of health utility changes and their validity and responsiveness in patients who had longstanding health complaints attributed to dental amalgam fillings is sparse. Thus, additional studies of the change in health utility outcomes that enable the calculation of quality-adjusted life year (QALY) and facilitate the comparison with other health conditions are warranted.

Health state utility (HSU) instruments consist of a descriptive system and a predetermined utility weight. Utilities are cardinal values that reflect an individual's preferences assigned to each possible combination of health states in the descriptive system $[4,5]$. They are measured on an interval scale with zero reflecting states of health equivalent to death and one reflecting full health. The EuroQol Five-Dimensional Questionnaire (EQ-5D) [6] and the Medical Outcomes Short-Form SixDimension (SF-6D) [7] are the most widely used HSU instruments. Such utilities are typically combined with survival estimates and aggregated across individuals to generate QALYs. QALYs are widely used as a measure of health outcomes in economic evaluations of health interventions. Economic evaluations play an increasing role in resource allocation decisions in healthcare, and it is important to critically assess the utility weights that form the basis for estimating QALY [8].

The EQ-5D has five dimensions, each with three severity levels in the original version (EQ-5D-3L) and five severity levels in the revised version (EQ-5D-5L). The revised version was designed to minimize the ceiling effect and improve the sensitivity of the $3 \mathrm{~L}$ version [9].
There are several country-specific EQ-5D-5L valuation studies that are currently ongoing (including the elicitation of Norwegian values) or already published [10]. The EQ-5D instrument is commonly recommended for economic evaluation by reimbursement agencies, such as the National Institute for Care and Excellence (NICE) in the United Kingdom (UK) [11] and the Norwegian Medicines Agency in Norway [12]. Despite the publication of the new English EQ-5D-5L value set, NICE's interimposition at the time of the analysis (December 2020) is that the validated cross-walk or mapping function by van Hout et al. [13] to derive value sets for the EQ-5D-5L from the existing $3 \mathrm{~L}$ version should be used for economic evaluation. Based on NICE's recommendation, the Norwegian Medicines Agency also use the cross-walk value sets by van Hout and colleagues for single technology assessments [12]. In general, EQ-5D is the most widely used HSU instrument in economic evaluation, followed by SF-6D [14, 15], and hence the focus of the present study.

The psychometric properties of EQ-5D and SF-6D have been widely investigated in different patient groups as well as in the general population. A recent systematic review of the literature demonstrated excellent psychometric properties of the EQ-5D across a broad range of populations and conditions [16]. The EQ-5D appears to be reliable and valid in the general population [17-22], and so does the SF-6D [23-25]. Thus, the reliability and validity of such instruments in the general population are useful for future population health studies. In addition to evidence of validity, both EQ-5D and SF-6D have been shown to be responsive in the general population, but the mean quality of life measured by EQ-5D is usually higher than that of SF-6D [26-28].

Furthermore, both EQ-5D and SF-6D have shown evidence of validity, and responsiveness for a number of diseases [29-36]. One important test of validity is the ability of a health outcome measure to reflect relevant changes in the health of patients over time, which, specifically, is referred to as responsiveness of a measure [37]. Although these instruments are becoming more common in clinical practice, the meaning of a change in score on such HSU instrument is not straightforward, mainly because of the unfamiliar units in the scale of these instruments [38]. These health outcome measures can be completed at baseline and at follow-up, and generate score changes, which allows us to easily calculate the statistical 
significance of the score changes. However, establishing the magnitude of the change score in a way easily understandable for health professionals, patients and policymakers has been difficult, though not impossible [38, 39]. Quantifying the magnitude of change that corresponds to a minimally important difference would help to address this problem [40]. From the patient perspective, Jaeschke et al. [41] defined the minimally important difference as "the smallest difference in score in the domain of interest which patients perceive as beneficial". Although we have some knowledge about validity and responsiveness of EQ-5D and SF-6D in some diseases [31, 35, 38, 42-44], we know very little about their responsiveness and validity in patients with MUPS who attribute their health problems to dental amalgam. To our knowledge, the minimally important difference of EQ-5D and SF-6D for amalgam patients has not been reported so far.

Therefore, we aimed to examine the health utility changes associated with dental amalgam fillings removal in a group of amalgam patients in terms of the two most widely used HSU instruments; the EQ-5D-5L and SF$6 D$. More specifically, this analysis had two objectives: (i) examine the concurrent and predictive validity as well as responsiveness to change of the EQ-5D-5L and SF-6D utilities in a prospective cohort study; and (ii) assess the ability of the EQ-5D-5L and SF-6D instruments to detect minimally important changes over time.

\section{Methods}

\section{Data and study design}

The analyses are based on data from a prospective cohort in Norway [45]. The study comprised three groups recruited separately: (i) patients with MUPS, which they attributed to dental amalgam restorations who wished to have their amalgam fillings removed (Amalgam cohort); (ii) patients with MUPS recruited from general practice without symptom attribution to amalgam fillings (MUPS cohort); and (iii) participants who identified themselves as healthy (Healthy cohort).

This analysis is restricted to the Amalgam cohort, which was the main target group. The presence of at least one amalgam fillings is the primary criteria for inclusion in amalgam cohort. Other inclusion criteria were unspecific health complaints attributed to dental amalgam restorations at least for three months, wish to have all amalgam fillings removed, ability to comply with the protocol, age between 20 and 70 years, permanent residence in Norway. Detailed eligibility criteria and recruitment procedures are reported elsewhere [45]. In general, a total of 49 participants were initially assessed for inclusion in the Amalgam cohort. Of these, 12 subjects did not fulfill the eligibility criteria and 5 did not complete the amalgam removal, and were excluded from the analysis.
A total of 32 participants were available for the follow-up analysis.

\section{Variables}

At baseline, the socio-economic variables age, gender, marital status, education, household income and employment status were documented. Patients also completed the EQ-5D-5L and the short-form 36 questionnaires (SF-36) at baseline and at follow-up (12 months after amalgam removal). Based on SF-36 questionnaire, we calculated the SF-6D utility [7]. Patients also rated their overall health on the visual analogue scale (EQ-VAS) and documented general symptoms in a health complaints index (GHC-index) questionnaire. The latter two (GHC and EQ-VAS) were used as the criteria variable against which the utility instruments were compared.

\section{Europol Five-Dimensional Questionnaire (EQ-5D-5L)}

At the time of this analysis, the psychometric property of the EQ-5D-5L was not yet studied in Norwegian population. However, a study of the Norwegian population norms for EQ-5D-3L [46] demonstrated that it can be used as reference data to compare patients with specific conditions and to assess the burden of the condition in question. The EQ-5D-5 $\mathrm{L}^{1}$ describes health along five dimensions: mobility, self-care, usual activities, pain/ discomfort and anxiety/depression. Each dimension is assessed by a single question on a five-point ordinal scale (no problems, slight problems, moderate problems, severe problems, extreme problems/unable to). Thus, the EQ-5D-5L defines $5^{5}=3125$ possible health states. These health states can be converted to a single EQ-5D-5L summary index by applying scores from a standard set of values (utility weights) derived from general population samples [47]. In the absence of Norwegian utility weights, we used utility weights that were derived from members of the English general public using composite time-trade-off [48]. We also tested the consistency of our results by applying the United Kingdom (UK) utility weights calculated using cross-walk algorithm (mapping the EQ-5D-5L descriptive system data onto the $3 \mathrm{~L}$ value set) from van Hout et al. [13]. The UK cross-walk value set is denoted as EQ-5D-CW to distinguish it from the directly elicited English value set, defined as EQ-5D-5L. The minimum value for the worst health state ("the pits") was -0.285 for the English EQ-5D-5L value set and -0.594 for the UK EQ-5D-CW.

The full health state, which is the absence of any problem on each of the 5-dimensions of the EQ-5D-5L

\footnotetext{
${ }^{1}$ EQ-5D-5L was available after registration at EuroQol Group http://www. euroqol.org.
} 
(11111), gives a utility of 1 . In contrast, the worst health state-which corresponds to level-5 on each dimension (55555); i.e., unable to walk about, unable to wash or dress myself, unable to do my usual activities, have extreme pain/discomfort, and extremely anxious/ depressed-produces a negative utility. The exact value of health state utilities vary depending on which country's value set (utility weight) has been applied [8]. In general, negative utilities imply that patients would prefer to die than live with such poor health states. Thus, utility is the preference for a health state (rated in the presence of choice) relative to full health (scored 1) and death (scored 0 ), and values below zero representing health states worse than being dead.

\section{The Short-Form Six-Dimension (SF-6D)}

The SF- $6 D^{2}$ is derived from 11 items of the SF-36 or SF-12 health survey, and has six-dimensions: physical functioning, role functioning, social functioning, bodily pain, mental health, and vitality [7, 49]. Each dimension has four to six severity levels, defining 18000 unique health states. Studies for the population norms of the SF-36 in the Norwegian general population supported the validity of the instrument $[50,51]$. Since there is no Norwegian-specific SF-6D value sets, the utility weights for SF-6D health states are based on members of the UK general population, and were elicited using standard gamble [7]. The maximum SF-6D utility is 1 , the minimum score for a living person (the worst state) is 0.296 , and the state being dead is scored as zero.

\section{EuroQol visual analogue scale (EQ-VAS)}

For the EQ-VAS, which is part of the EQ-5D-5L questionnaire, patients are asked to indicate their overall health on a vertical visual analogue scale, ranging from 0 (worst imaginable health) to 100 (best imaginable health). EQ-VAS can be used to measure a multitude of subjective conditions and would seem particularly appropriate for conditions related to MUPS.

\section{General health complaints (GHC-index)}

The GHC-index is the sum score of 12 items, each scored by use of numeric rating scales ranging from 0 to 10 [52]. The items are: Musculoskeletal complaints, gastrointestinal complaints, cardiovascular complaints, skin problems, complaints related to eyes/sight, complaints related to ears/hearing/nose/throat, tiredness, dizziness, headaches, memory problems, difficulty concentrating, and anxiety/depression. We reversed the score values $(\mathrm{GHCr})$

\footnotetext{
${ }^{2}$ SF-6D was available after registering at University of Sheffield https://licen sing.sheffield.ac.uk/product/SF-6D.
}

to obtain a scale in which higher scores represent better health (i.e., less health complaints) and positive change scores represented improvement. The GHC-index is not utility-weighted, and the index value is the crude sum of scores over the 12 dimensions with a maximum value of 120.

\section{Statistical analyses \\ Concurrent and predictive validity}

Concurrent validity of each of the two HSU instruments was tested by computing Spearman's rank correlation coefficients $(r h o, \rho)$ between the utility instruments and each of the criterion measures at the baseline. A non-parametric Spearman's rank correlation coefficient was chosen based on the measures' score distributions. Because health is not a static variable, we repeated the concurrent validity analyses in the follow-up period.

Predictive validity refers to the association between one variable and an outcome assessed at a later time [53, 54]. In this study, predictive validity was calculated as the correlation $(\rho)$ between the HSU instruments at baseline and the criterion measures at follow-up. The strength of the relationship was considered low/weak $(\rho<0.25)$, fair $(\rho=0.25-0.50)$, good $(\rho=0.50-0.75)$, and excellent $(\rho>0.75)[16]$.

\section{Responsiveness}

Responsiveness is a measure of the sensitivity of an instrument to change in health status over time. First, changes in all health outcome measures at the baseline and follow-up were compared using paired $t$-tests. Second, responsiveness was assessed using effect size (ES), standardized response mean (SRM) and relative efficiency (RE). ES was defined as the mean observed change from baseline to follow-up divided by the standard deviation of the baseline score [55]. SRM was calculated in the same way as the ES, but using the standard deviation of the pre-post differences as denominator. ES and SRM were classified as large $(\geq 0.8)$, moderate $(0.5-0.79)$ or small $(<0.5)[56,57]$. Both ES and SRM are standardized measures of change over time in health, independent of sample size [58]. RE was calculated by taking a ratio of $F$-statistics (or squared $t$-statistics), where the criterion measure served as the reference. An RE value less than 1 implies that the standard criterion measure is more responsive than the utility instruments, and the converse is true for an RE value of greater than 1 . A coefficient of 1 indicates similarity in the efficiency of the two measures.

\section{Minimally important change}

Minimally important change (MIC) is defined as the smallest change in score which is perceived as important by patients or clinicians [59]. In this study, MIC 
was assessed using both distribution and anchor-based approaches. Distribution-based methods mainly measure minimally detectable change (the smallest change that can be detected by the instrument beyond measurement error) [60]. Nonetheless, the term MIC is used for both approaches in this paper. For distribution-based calculations, MIC was defined as half baseline standard deviation $\left(0.5^{*} \mathrm{SD}\right)$ for the effectiveness of the intervention [61]. For the anchor-based method, MIC is usually estimated by comparing change scores with an external anchor. One of the commonly used anchors for establishing MIC is global ratings of change. For the present analysis, we used patients' self-reported global ratings of results of amalgam fillings removal as external anchor. Participants were asked "How do you rate the results of the amalgam fillings removal?" The pre-defined responses were:

1. Fully recovered.

2. Much better.

3. Somewhat better.

4. No change.

5. Worse.

The relationship between the global ratings of change question and changes in EQ-5D-5L and SF-6D utilities was assessed by calculating the change in EQ-5D-5L and SF-6D utilities from baseline to follow-up for each patient, and likewise for the GHCr-index and EQ-VAS. In accordance with previous studies, we considered patients whose global ratings of change score was 3 or 5 as having experienced some change equivalent to the MIC [38, 62]. For participants who reported a worsening of health (rating scale of 5), the sign of the change in each health outcome measure was reversed. The MIC was then taken as the mean changes in scores of the patients who scored 3 or 5 .

Finally, we applied predictive modelling, which is a newly proposed anchor-based method for MIC [63]. Here, Item-2 of the SF-36 was used as the anchor, which is described as: "Compared to one year ago, how would you rate your health in general now?".

1. Much better now than 1 year ago.

2. Somewhat better now than 1 year ago.

3. About the same.

4. Somewhat worse now than 1 year ago.

5. Much worse now than 1 year ago.

This score was transformed into a change score by taking the difference between baseline and follow-up to produce an anchor. Then, we used exact logistic regression to predict whether a patient belongs to the improved $(\geq 1)$ or not improved group $(\leq 0)$ on this anchor using
Table 1 Baseline sample characteristics for patients with MUPS undergoing amalgam removal

\begin{tabular}{ll}
\hline Characteristics & N (\%) \\
\hline Female gender & $19(59.4)$ \\
Education & \\
Lower \& upper secondary & $14(43.8)$ \\
College, < 4 years & $11(34.4)$ \\
College, 4 +years & $7(21.9)$ \\
Living with partner & \\
No & $6(18.7)$ \\
Yes & $26(81.3)$ \\
Income & \\
Low & $9(25.8)$ \\
Middle income & $13(41.9)$ \\
High income & $10(32.3)$ \\
\hline MUPS medically unexplained physical symptoms &
\end{tabular}

the change in each of the health outcome measure as the predictor. When sample sizes are small or the data are skewed, exact conditional inference is often more appropriate compared to the conventional method of logistic regression [64]. The MIC was estimated from the equation: $M I C=\left[\ln \left(\operatorname{Odds}_{\text {Pre }}\right)-\mathrm{C}\right] / \beta$, where $\mathrm{C}$ is the intercept, $\beta$ is the regression coefficient, and Odds $s_{\text {Pre }}$ is pre-odds of being improved and given by $\mathrm{P} /(1-\mathrm{P})$, with $\mathrm{P}$ the proportion improved based on the anchor [63].

To confirm the usefulness of the anchors, Spearman's rank correlations were computed between the health outcome score changes and the two anchors. A correlation coefficient of 0.30 or more is considered sufficiently strong to allow for computation of MIC [65].

\section{Results}

Baseline characteristics

Demographic and socio-economic characteristics are presented in Table 1. Most patients were female (59.4\%), with the majority living with a partner or spouse (81.3\%). Mean age of patients at baseline was $52(\mathrm{SD}=7.5)$ years. Boxplots illustrate that the distributions were wide for all five health outcomes measures used, both at baseline and at follow-up (Fig. 1). No patient reported either the worst or best possible health on any of the health outcome measures.

\section{Concurrent and predictive validity}

Spearman's correlation coefficients for the HSU instruments and the criteria measures (GHCr-index and EQVAS) at baseline and follow-up periods are presented in Table 2. For the EQ-5D-5L, the correlations with GHCrindex were relatively lower at baseline $(\rho=0.48)$ than at follow-up $(\rho=0.52)$, with a reverse pattern for EQ-VAS 
a

Distributions of HSU instruments
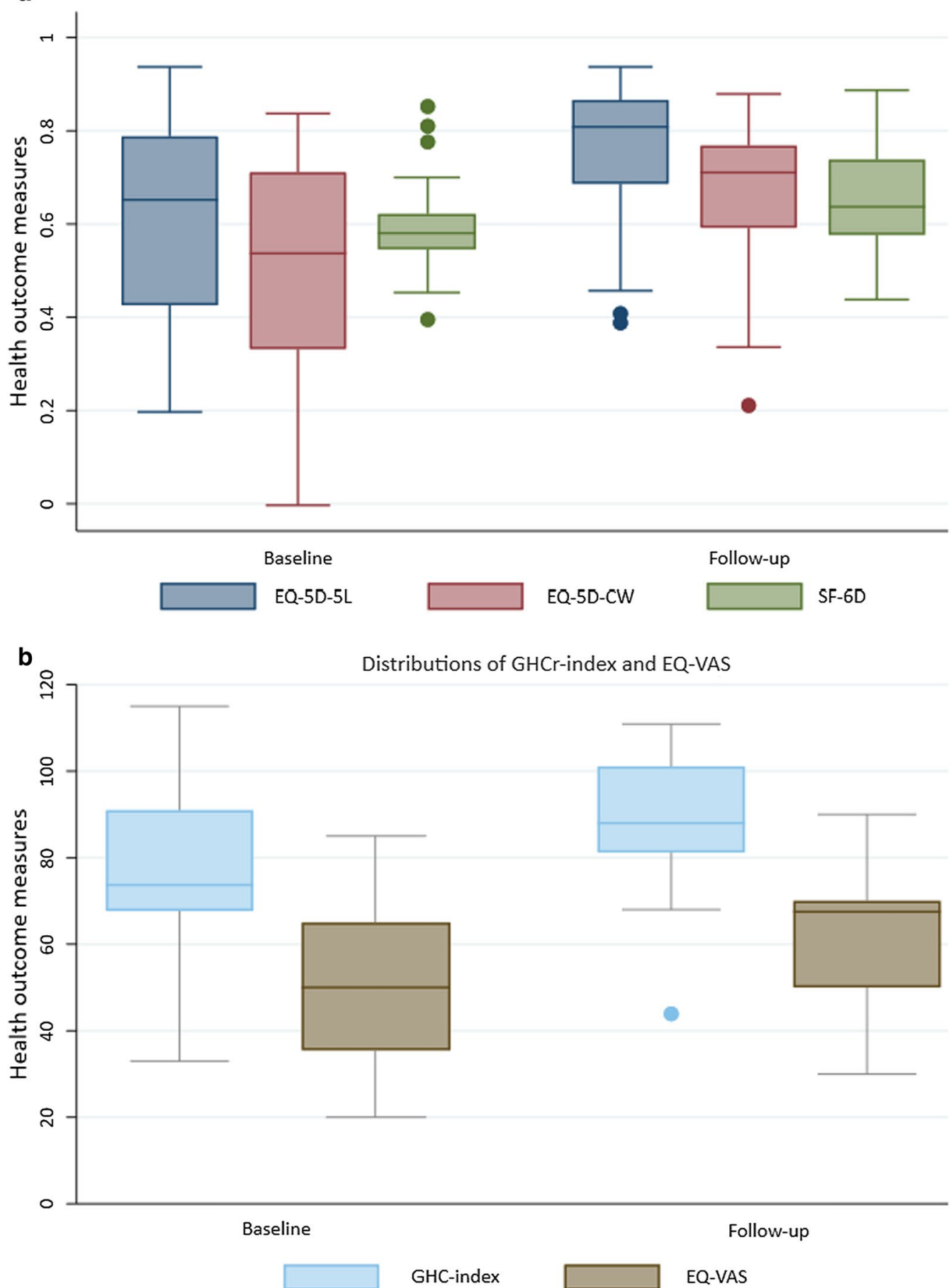

Fig. 1 Box plots showing distributions of health outcomes at the baseline and follow-up for amalgam patients. A box indicates the positions of the upper and lower quartiles; the interior of the box indicates the interquartile range; the crossbar (middle line) that intersects the box shows the median of the dataset; a whisker (line) that extends to the extreme of the distribution from lower hinge and upper hinge indicates the minimum and maximum values, respectively. EQ-5D-5L EuroQol 5-dimensional 5-level questionnaire; EQ-5D-CW EQ-5D cross-walk value set; SF-6D Short-form 6-dimension; GHCr (reversed) general health complaints; EQ-VAS (EuroQol) visual analogue scale; HSU Health state utility 
Table 2 Correlations as measures of concurrent and predictive validities for EQ-5D-5L and SF-6D utilities

\begin{tabular}{|c|c|c|c|}
\hline & EQ-5D-5L & EQ-5D-CW & SF-6D \\
\hline \multicolumn{4}{|c|}{ Concurrent validity } \\
\hline \multicolumn{4}{|l|}{ Baseline } \\
\hline GHCr-index & 0.48 & 0.46 & 0.42 \\
\hline EQ-VAS & 0.74 & 0.68 & 0.70 \\
\hline \multicolumn{4}{|l|}{ Follow-up } \\
\hline GHCr-index & 0.52 & 0.56 & 0.50 \\
\hline EQ-VAS & 0.55 & 0.58 & 0.64 \\
\hline \multicolumn{4}{|c|}{ Predictive validity $^{a}$} \\
\hline GHCr-index & 0.25 & 0.22 & 0.31 \\
\hline EQ-VAS & 0.31 & 0.28 & 0.46 \\
\hline
\end{tabular}

a Correlations between utility instruments at baseline and criteria variables at follow-up, which are classified as weak $(\rho<0.25)$, fair $(0.25$ to 0.50$)$, good ( 0.50 to 0.75 ), and excellent (>0.75). EQ-5D-5L EuroQol 5-dimensional 5-level questionnaire; EQ-5D-CW EQ-5D cross-walk value set; SF-6D Short-form 6-dimension; $\mathrm{GHCr}$ (reversed) general health complaints; EQ-VAS, (EuroQol) visual analogue scale

(higher at baseline). The results were quite similar for the EQ-5D-CW and the SF-6D. These correlations were generally high, indicating good concurrent validities of the HSU instruments. With regard to predictive validity, the EQ-5D-5L and SF-6D at the baseline period predicted both the GHCr-index and EQ-VAS at follow-up period fairly well, with SF-6D performing slightly better. The EQ-5D-CW fairly predict EQ-VAS, but slightly week in predicting $\mathrm{GHCr}$-index.

\section{Responsiveness and minimally detectable change}

Responsiveness results are presented in Table 3. The baseline means (SD) EQ-5D-5L and SF-6D utilities were 0.61 $(0.22)$ and $0.60(0.10)$, respectively. Mean EQ-5D-CW was 0.10 lower than mean EQ-5D-5L both in the baseline and follow-up. All health outcome measures showed significant improvement at follow-up $(p<0.01$, paired $t$-tests). The mean change (SD) for EQ-5D-5L was 0.158 $(0.207)$ and that of SF-6D was $0.056(0.084)$. The mean change for the EQ-5D-CW is quite like the EQ-5D-5L. The corresponding values for $\mathrm{GHCr}$-index and EQ-VAS were 12.78 (15.87) and 12.53 (15.23), respectively.

Generally, all health outcome measures exhibited moderate responsiveness as measured by ES and SRM. Except the SF-6D, all health outcome measures revealed similar ES $(\approx 0.70)$. The SF-6D utility instrument had relatively lower responsiveness $(E S=0.58)$ (Table 3$)$. In terms of SRM, both criteria variables revealed large responsiveness $($ SRM $>0.80)$, while the two utility instruments indicated moderate responsiveness, as expected. All HSU instruments had RE below 1, indicating less efficiency in detecting changes in health over time as compared to $\mathrm{GHCr}$-index (though statistically insignificant, except SF-6D). Both EQ-5D-5L and EQ-5D-CW were more efficient when compared with SF-6D.

MIC estimations derived from the anchor- and distribution-based methods, as well as the correlations between health outcome score changes and the two anchors are presented in Table 4. All health outcome score changes were significantly correlated with responses to both anchors; accordingly, the two global ratings of change were considered appropriate anchors for measuring all score changes.

For the anchor-based method, the MIC estimation was 0.118 for the EQ-5D-5L, 0.124 for EQ-5D-CW and 0.064 for SF-6D. The corresponding MIC was 9.158 for the GHCr-index and 8.789 for the EQ-VAS score. The distribution-based estimation (i.e., half SD of baseline) gave 0.108 for the EQ-5D-5L, 0.115 for EQ-5D-CW and 0.048 for the SF-6D. Both the distribution- and anchor-based estimations produced fairly similar results. The predictive modelling approach also produced similar findings, confirming the consistency of our results.

\section{Discussion}

In this analysis of patients with health complaints attributed to their amalgam fillings, validity and responsiveness to change from baseline to follow-up (12 months after removal of amalgam fillings) was assessed. This study is

Table 3 Measures of responsiveness for health outcome measures

\begin{tabular}{|c|c|c|c|c|c|c|c|}
\hline & \multicolumn{2}{|l|}{ Mean (SD) } & \multicolumn{2}{|l|}{ Paired t-test } & \multicolumn{3}{|c|}{ Responsiveness } \\
\hline & Baseline & Follow-up & $\Delta$ Mean (SD) & $p$ value & ES (SE) & SRM (SE) & $\mathrm{RE}(\mathrm{SE})$ \\
\hline EQ-5D-5L & $0.61(0.22)$ & $0.77(0.16)$ & $0.158(0.207)$ & $<0.001$ & $0.73(0.17)$ & $0.76(0.18)$ & $0.90(0.58)$ \\
\hline EQ-5D-CW & $0.51(0.23)$ & $0.67(0.17)$ & $0.162(0.220)$ & 0.002 & $0.70(0.16)$ & $0.75(0.19)$ & $0.84(0.57)$ \\
\hline SF-6D & $0.60(0.10)$ & $0.65(0.12)$ & $0.056(0.084)$ & 0.001 & $0.58(0.19)$ & $0.67(0.23)$ & $0.68(0.34)$ \\
\hline GHCr-index ${ }^{a}$ & $76.72(17.75)$ & $89.49(14.36)$ & $12.78(15.87)$ & $<0.001$ & $0.72(0.19)$ & $0.81(0.18)$ & 1 (Ref.) \\
\hline EQ-VAS & $49.90(18.21)$ & $62.44(16.72)$ & $12.53(15.23)$ & $<0.001$ & $0.69(0.17)$ & $0.82(0.19)$ & $1.05(0.48)$ \\
\hline
\end{tabular}

a $\mathrm{GHCr}$-index has been used as a reference in the calculation of RE. $\triangle$ Mean Mean change between baseline and follow-up; $S D$ standard deviation; $S E$ bootstrapped standard error (with 1000 iterations); ES effect size; SRM standardized response mean; RE relative efficiency; EQ-5D-5L EuroQol 5-dimensional 5-level questionnaire; EQ-5D-CW EQ-5D cross-walk; SF-6D Short-form 6-dimension; GHCr (reversed) general health complaints; EQ-VAS (EuroQol) visual analogue scale 
Table 4 Minimally important changes for those who reported some change

\begin{tabular}{|c|c|c|c|c|c|c|c|}
\hline & \multicolumn{2}{|c|}{ Correlations $(\rho)^{\mathrm{a}}$} & \multirow{2}{*}{$\begin{array}{l}0.5 \mathrm{SD} \\
\mathrm{MIC}\end{array}$} & \multicolumn{2}{|c|}{ Anchor-based method } & \multicolumn{2}{|c|}{ Predictive modelling } \\
\hline & GRC1 & GRC2 & & MIC & $95 \% \mathrm{Cl}$ & $\mathrm{MIC}$ & $95 \% \mathrm{Cl}$ \\
\hline EQ-5D-5L & 0.53 & 0.49 & 0.108 & 0.118 & $0.038-0.198$ & 0.103 & $0.062-0.237$ \\
\hline EQ-5D-CW & 0.52 & 0.45 & 0.115 & 0.124 & $0.036-0.213$ & 0.101 & $0.018-0.325$ \\
\hline SF-6D & 0.45 & 0.51 & 0.048 & 0.064 & $0.029-0.099$ & 0.056 & $0.020-0.143$ \\
\hline GHCr-index & 0.36 & 0.31 & 8.876 & 9.158 & $1.798-16.517$ & 8.786 & $3.096-17.804$ \\
\hline EQ-VAS & 0.45 & 0.36 & 9.106 & 8.789 & $2.580-14.999$ & 9.076 & $3.525-18.624$ \\
\hline
\end{tabular}

a Correlations of GRC variables with score changes of utility and criteria variables (a value of $\rho 0.30$ and above is sufficiently large to use the anchors for the calculation of MIC). GRC Global rating of change ( 1 = evaluation of amalgam removal results, \& $2=$ item-2 of the SF-36); Cl (bootstrapped) confidence interval (with 1000 iterations); SD (baseline) standard deviation; MIC minimally important change; EQ-5D-5L EuroQol 5-dimensional 5-level questionnaire; SF-6D Short-form 6-dimension; $\mathrm{GHCr}$ (reversed) general health complaints; EQ-VAS (EuroQol) visual analogue scale

the first to assess the validity and responsiveness of two commonly used utility instruments-the EQ-5D-5L and SF-6D-in patients with MUPS who attribute their health problems to amalgam fillings. Our results have shown key differences in the ability of the EQ-5D-5L and SF-6D to measure longitudinal changes. Although both EQ-5D-5L and SF-6D demonstrated significant change in health over time, the EQ-5D-5L was more responsive to change than the SF-6D. This finding is in line with previous studies of the two utilities in other health conditions $[31,38,66]$.

The correlation between the utility and criterion measures showed fair concurrent validity at both baseline and follow-up. The high correlations between criteria measures and the two HSU instruments generally demonstrated acceptable concurrent validity of EQ-5D-5L and SF-6D. At the end of the follow-up period, correlations were increased between the SF-6D and criterion measures unlike EQ-5D-5L. The main explanation could be that SF-6D captures most of the description of the more specific and comprehensive scale of $\mathrm{GHCr}$-index and EQ-VAS compared to the EQ-5D-5L. For instance, both SF-6D and GHCr-index focus on physical functioning and energy, which the EQ-5D-5L lacks. This implies that the intervention was followed by reduction of intensity of the health complaints, increasing its consistency with the more specific and comprehensive scales of criteria measures [31].

Further, our findings showed that EQ-5D-5L and SF-6D had sufficient predictive validity - the utility instruments at the baseline would fairly predict the GHCr-index at follow-up, indicating that they accounted for a significant amount of variance in predicting amalgam treatment outcomes at follow-up. The predictive power may depend on the severity of the condition, which could not be investigated due to small sample size. However, regression to the mean could partly be considered as a possible cause of an observed change, because regression to the mean in repeated data is a ubiquitous phenomenon $[67,68]$.
With regard to responsiveness, our results generally showed moderate to large responsiveness for all the health outcome measures. The EQ-5D-5L and SF-6D were moderately responsive to changes based on both the ES and SRM statistics, whereas the EQ-VAS and $\mathrm{GHCr}$-index were highly responsive to changes on the SRM and relative efficiency statistics. Other studies in different diseases found similar results $[31,66]$. The high responsiveness and greater efficiency would suggest that GHCr-index and EQ-VAS are suitable criteria measures for amalgam patients. However, a firm conclusion that the GHCr-index and EQ-VAS are more responsive than the HSU instruments as a measure of health in amalgam patients would be premature, because the findings are based on an analysis with a small sample size.

To our knowledge, empirical work has not been performed to assess the responsiveness and MIC of the EQ5D-5L and the SF-6D in patients with MUPS attributed to amalgam fillings. Both the anchor-based and distribution-based approaches have shown similar MIC values. While the MIC values calculated by the distributionand anchor-based methods were fairly similar for the EQ-VAS and the GHCr-index, a substantial difference was observed between the EQ-5D-5L and SF-6D. The MIC difference between the two utility instruments was mainly attributable to their scale difference. For instance, the effective scale length for the English EQ-5D-5L is 1.285 (i.e., from -0.285 to 1 ), whereas the effective scale length for the SF-6D is 0.704 (i.e., 0.296 to 1 ). The scale adjusted anchor-based MIC for EQ-5D-5L was 0.092 $(=0.118 / 1.285)$, which is equivalent to that of SF-6D $(0.091=0.064 / 0.704)$. Similar results were observed for the distribution-based MIC. Thus, the scale difference mainly accounts for the difference in their MIC values. Our finding is consistent with previous studies for EQ5D-3L and SF-6D [38]. This implies that it is not only the description of health but also the range of the instrument scale that is crucial in the assessment of MIC for the HSU instruments. While EQ-5D-5L applied time-trade-off for 
elicitation of utility weights, SF-6D used standard gamble. Thus, their difference arises primarily because of scale effect brought up by the methodological approach used to construct preference weights [69].

The EQ-5D-5L and SF-6D are designed to be utilized to calculate QALYs (measured in units of time) for the estimation of cost-effectiveness. Therefore, the validity of the instruments will translate into the validity for the costeffectiveness estimates. Usually, the EQ-5D-5L tends to produce larger change scores than the SF-6D and hence produces more favorable cost-effectiveness ratios than the SF-6D, especially when baseline health was strongly compromised as the case in the present study [70]. Similarly, other studies found higher QALY gains using the EQ-5D-3L than the SF-6D [33, 36, 71, 72].

Both HSU instruments had good concurrent validity and fair predictive validity in patients with amalgam removal. While EQ-5D consistently showed better responsiveness, SF-6D had slightly better predictive power. The results also depend on the choice of the criteria variables and the disease conditions in question. Being more responsive, the EQ-5D could be more appropriate for measuring the burden of health conditions or for generating QALYs that can be used in economic evaluation studies than the SF-6D in line with previous study [73]. Nevertheless, both HSU instruments are valid economic evaluation instruments but not interchangeable and hence, the choice of HSU instrument for measuring utility can lead to different results in the context of cost per QALY estimates. This suggests that researchers and policy makers using cost-effectiveness analysis must consider several sources of evidence to select an instrument for measuring utility. Since there is no gold standard, decision makers need to consider an instrument that enable them to make consistent decisions across a broad range of populations and conditions. Further research is required, particularly across the full severity range of the utility scale, to identify the practical performance of utility instruments and their implications for cost-per-QALY estimates and health care decision making.

The main strength of this study is that it applied several techniques to validate instruments to elicit health outcome measures, including ES, SRM and relative efficiency as well as mean change scores to measure the responsiveness, which enables us to confirm the consistency of our results. Further, we applied distribution-based and multiple anchor-based assessment of MIC for the EQ-5D-5L and SF-6D that substantiate the stability of our findings. Although there is no single gold standard external criterion, anchor-based techniques rely most commonly on the use of a subjective global assessment of change [74], which have the advantage of linking the change in a given score to the patient's perspective [58].

This study has also a number of limitations. Because global assessment is based on the recall ability of patients about their earlier health status, the use of the retrospective global assessment as an external criterion of score changes can be problematic. That is, there could be a potential for response shift and recall bias due to the prolonged time between the baseline and follow-up periods [38]. Thus, the MIC may change over time and recall bias and response shift may pose a problem, which needs to be investigated further. Furthermore, only a few patients reported deterioration and, hence, we did not analyse MIC for the clinically deteriorated patients separately. The small sample size also precluded the use of subgroup analyses. Thus, although this study has used several methods to quantify MIC, it is important to further test and validate estimates using other methods and larger sample sizes.

\section{Conclusions}

In conclusion, the concurrent and predictive validity from all health outcomes were acceptable. The discrepancy in responsiveness of EQ-5D-5L and SF-6D in detecting change was mainly attributed to their scale differences. Thus, both EQ-5D-5L and the SF-6D can be used in clinical trials including this group of patients where a known effective intervention is to be applied. The MIC estimate for the EQ-5D-5L and SF-6D will be useful in interpreting EQ-5D-5L and SF-6D utilities, both in individuals and in groups of patients participating in trials as well as in the planning of new trials. The differences in the magnitude of the absolute change scores have important implications for cost-effectiveness analyses. Economic evaluation studies should be based on health utilities elicited with instruments that have validated measurement properties for the intended population.

\section{Abbreviations}

EQ-5D (5L/3L/CW): EuroQol 5-Dimensional (5-Level/3-level/Crosswalk); EQ-VAS: EuroQol Visual Analogue Scale; ES: Effect Size; GHCr: General Health Compliant (reversed); GRC: Global rating of change; HSU: Health State Utility; MIC: Minimally Important Change; MUPS: Medically Unexplained Physical Symptoms; NICE: National Institute for Care and Excellence; QALY: QualityAdjusted Life Year; RE: Relative Efficiency; $\rho$ : Spearman's rank correlation coefficient (rho); SD: Standard Deviation; SE: Standard Error; SF-6D: Short Form 6-Dimension; SF-36: Short Form 36 (questionnaire); SRM: Standardized Response Mean; UK: United Kingdom.

\section{Acknowledgements}

Not applicable. 


\section{Authors' contributions}

Study conception and design: ANL; LB; TA; HH; FM; BR. Acquisition of data: LB. Analysis and interpretation of data: ANL; LB; BR. Drafting of manuscript: ANL. Critical revision and editing: $A N L ; L B ; F M ; T A ; H H ; B R$. All authors approved the final manuscript.

\section{Funding}

The study was funded by Norwegian Ministry of Health and Care Services via the Norwegian Directorate of Health. The funder has no role in the design of the study, statistical analyses, or interpretation of the results.

\section{Availability of data and materials}

The datasets generated and analysed during the current study are not publicly available due to privacy concern as relatively few patients participated in the study with implications for potential identification through personal characteristics.

\section{Declarations}

\section{Ethics approval and consent to participate}

The trial was approved by the local research ethics committee (REK2012/331) and registered at ClinicalTrials.gov (https://clinicaltrials.gov/ct2/show/NCT01 6822782. Written informed consent was obtained from all participants in this study. All procedures performed in studies involving human participants were in accordance with the ethical standards of the institutional and/or national research committee and with the 1964 Helsinki declaration and its later amendments or comparable ethical standards.

\section{Consent for publication}

Not applicable.

\section{Competing interests}

The authors declare that they have no competing interests.

\section{Author details}

${ }^{1}$ Section for Ethics and Health Economics, Department of Global Public Health and Primary Care, Faculty of Health Sciences, University of Bergen, 5020 Bergen, Norway. ${ }^{2}$ Dental Biomaterials Adverse Reaction Unit, NORCE Norwegian Research Centre AS, Bergen, Norway. ${ }^{3}$ Department of Clinical Dentistry, University of Bergen, Bergen, Norway. ${ }^{4}$ Institute for Applied Epistemology and Medical Methodology, University of Witten/Herdecke, Freiburg, Germany. ${ }^{5}$ National Research Center in Complementary and Alternative Medicine, NAFKAM, Department of Community Medicine, UiT The Arctic University of Norway, Troms $\varnothing$, Norway.

Received: 25 November 2020 Accepted: 3 April 2021

Published online: 17 April 2021

\section{References}

1. Bates MN. Mercury amalgam dental fillings: an epidemiologic assessment. Int J Hyg Environ Health. 2006;209(4):309-16.

2. Kristoffersen AE, Musial F, Hamre HJ, Björkman L, Stub T, Salamonsen A, Alræk T. Use of complementary and alternative medicine in patients with health complaints attributed to former dental amalgam fillings. BMC Complement Altern Med. 2016;16:22.

3. Melchart D, Vogt S, Kohler W, Streng A, Weidenhammer W, Kremers L, Hickel R, Felgenhauer N, Zilker T, Wuhr E, Halbach S. Treatment of health complaints attributed to amalgam. J Dent Res. 2008;87(4):349-53.

4. Drummond MF, Sculpher MJ, Claxton K, Stoddart GL, Torrance GW. Methods for the economic evaluation of health care programmes. Oxford: Oxford University Press; 2015.

5. Whitehead SJ, Ali S. Health outcomes in economic evaluation: the QALY and utilities. Br Med Bull. 2010;96(1):5-21.

6. Brooks R. EuroQol: the current state of play. Health Policy. 1996;37(1):53-72.
7. Brazier J, Roberts J, Deverill M. The estimation of a preference-based measure of health from the SF-36. J Health Econ. 2002;21(2):271-92.

8. Olsen JA, Lamu AN, Cairns J. In search of a common currency: a comparison of seven EQ-5D-5L value sets. Health Econ. 2018;27(1):39-49.

9. Herdman M, Gudex C, Lloyd A, Janssen M, Kind P, Parkin D, Bonsel G, Badia X. Development and preliminary testing of the new five-level version of EQ-5D (EQ-5D-5L). Qual Life Res. 2011:20(10):1727-36.

10. EuroQol. EQ-5D-5L Valuation: Standard value sets. The EuroQol Group. 2020. https://euroqol.org/eq-5d-instruments/eq-5d-5l-about/valuationstandard-value-sets/. Accessed 15 Nov 2020.

11. NICE. Methods for the development of NICE Public Health Guidance: NICE process and methods guides. 3rd ed. London: National Institute for Health and Care Excellence (NICE); 2012.

12. NoMA. Guidelines for the submission of documentation for single technology assessment (STA) of pharmaceuticals. Oslo: The Norwegian Medicines Agency (NoMA). 2020. https://legemiddelverket.no/. Accessed 10 June 2020 .

13. van Hout B, Janssen MF, Feng Y-S, Kohlmann T, Busschbach J, Golicki D, Lloyd A, Scalone L, Kind P, Pickard AS. Interim scoring for the EQ5D-5L: mapping the EQ-5D-5L to EQ-5D-3L value sets. Value Health. 2012;15(5):708-15.

14. Kennedy-Martin M, Slaap B, Herdman M, van Reenen M, KennedyMartin T, Greiner W, Busschbach J, Boye KS. Which multi-attribute utility instruments are recommended for use in cost-utility analysis? A review of national health technology assessment (HTA) guidelines. Eur J Health Econ. 2020;21(8):1245-57.

15. WisløffT, Hagen G, Hamidi V, Movik E, Klemp M, Olsen JA. Estimating QALY gains in applied studies: a review of cost-utility analyses published in 2010. Pharmacoeconomics. 2014;32(4):367-75.

16. Feng Y-S, Kohlmann T, Janssen MF, Buchholz I. Psychometric properties of the EQ-5D-5L: a systematic review of the literature. Qual Life Res. 2020;30:647-73.

17. Feng Y, Devlin N, Herdman M. Assessing the health of the general population in England: how do the three- and five-level versions of EQ-5D compare? Health Qual Life Outcomes. 2015;13:171.

18. Hernandez G, Garin O, Pardo Y, Vilagut G, Pont À, Suárez M, Neira M, Rajmil L, Gorostiza I, Ramallo-Fariña Y, et al. Validity of the EQ-5D-5L and reference norms for the Spanish population. Qual Life Res. 2018;27(9):2337-48

19. Hinz A, Kohlmann T, Stöbel-Richter Y, Zenger M, Brähler E. The quality of life questionnaire EQ-5D-5L: psychometric properties and normative values for the general German population. Qual Life Res. 2014;23(2):443-7.

20. Młyńczak K, Golicki D. Validity of the EQ-5D-5L questionnaire among the general population of Poland. Qual Life Res. 2020;30:817-29.

21. Craig BM, Pickard AS, Lubetkin El. Health problems are more common, but less severe when measured using newer EQ-5D versions. J Clin Epidemiol. 2014;67(1):93-9.

22. Agborsangaya CB, Lahtinen M, Cooke T, Johnson JA. Comparing the EQ-5D $3 \mathrm{~L}$ and 5L: measurement properties and association with chronic conditions and multimorbidity in the general population. Health Qual Life Outcomes. 2014;12(1):74.

23. Campolina AG, López RVM, Nardi EP, Ferraz MB. Internal consistency of the SF-6D as a health status index in the Brazilian urban population. Value Health Reg Issues. 2018;17:74-80.

24. Luo N, Wang P, Fu AZ, Johnson JA, Coons SJ. Preference-based SF-6D scores derived from the SF-36 and SF-12 have different discriminative power in a population health survey. Med Care. 2012;50(7):627-32.

25. Baxter S, Sanderson K, Venn A, Otahal P, Palmer AJ. Construct validity of SF-6D health state utility values in an employed population. Qual Life Res. 2015:24(4):851-70

26. Kangwanrattanakul K. A comparison of measurement properties between UK SF-6D and English EQ-5D-5L and Thai EQ-5D-5L value sets in general Thai population. Expert Rev Pharmacoecon Outcomes Res. 2020;1-10.

27. Shiroiwa T, Fukuda T, Ikeda S, Igarashi A, Noto S, Saito S, Shimozuma K. Japanese population norms for preference-based measures: EQ-5D-3L, EQ-5D-5L, and SF-6D. Qual Life Res. 2016;25(3):707-19. 
28. Zhao L, Liu X, Liu D, He Y, Liu Z, Li N. Comparison of the psychometric properties of the EQ-5D-3L and SF-6D in the general population of Chengdu city in China. Medicine. 2019;98(11):e14719.

29. Brazier J, Connell J, Papaioannou D, Mukuria C, Mulhern B, Peasgood T, Jones ML, Paisley S, O'Cathain A, Barkham M, et al. A systematic review, psychometric analysis and qualitative assessment of generic preferencebased measures of health in mental health populations and the estimation of mapping functions from widely used specific measures. Health Technol Assess. 2014;18(34):1-188.

30. Brazier J, Roberts J, Tsuchiya A, Busschbach J. A comparison of the EQ-5D and SF-6D across seven patient groups. Health Econ. 2004:13(9):873-84

31. Chen P, Lin KC, Liing RJ, Wu CY, Chen CL, Chang KC. Validity, responsiveness, and minimal clinically important difference of EQ-5D-5L in stroke patients undergoing rehabilitation. Qual Life Res. 2016;25(6):1585-96.

32. Engel L, Bryan S, Evers SMAA, Dirksen CD, Noonan VK, Whitehurst DGT. Exploring psychometric properties of the SF-6D, a preference-based health-related quality of life measure, in the context of spinal cord injury Qual Life Res. 2014;23(8):2383-93.

33. Goodwin PC, Ratcliffe J, Morris J, Morrissey MC. Using the knee-specific Hughston Clinic Questionnaire, EQ-5D and SF-6D following arthroscopic partial meniscectomy surgery: a comparison of psychometric properties. Qual Life Res. 2011;20(9):1437-46.

34. Khanna D, Furst DE, Wong WK, Tsevat J, Clements PJ, Park GS, Postlethwaite AE, Ahmed M, Ginsburg S, Hays RD, Ron D. Hays for the Scleroderma Collagen Type 1 Study Group. Reliability, validity, and minimally important differences of the SF-6D in systemic sclerosis. Qual Life Res. 2007:16(6):1083-92.

35. Mulhern B, Meadows K. The construct validity and responsiveness of the EQ-5D, SF-6D and Diabetes Health Profile-18 in type 2 diabetes. Health Qual Life Outcomes. 2014;12:42.

36. Obradovic $\mathrm{M}$, Lal $\mathrm{A}$, Liedgens $\mathrm{H}$. Validity and responsiveness of EuroQol-5 dimension (EQ-5D) versus Short Form-6 dimension (SF-6D) questionnaire in chronic pain. Health Qual Life Outcomes. 2013;11:110.

37. Glossary. Health outcomes methodology. Med Care. 2000;38(9):I17-13.

38. Walters SJ, Brazier JE. Comparison of the minimally important difference for two health state utility measures: EQ-5D and SF-6D. Qual Life Res. 2005;14(6):1523-32.

39. Hagg O, Fritzell P, Nordwall A. The clinical importance of changes in outcome scores after treatment for chronic low back pain. Eur Spine J. 2003;12(1):12-20

40. Juniper EF, Guyatt GH, Willan A, Griffith LE. Determining a minimal important change in a disease-specific Quality of Life Questionnaire. J Clin Epidemiol. 1994;47(1):81-7.

41. Jaeschke R, Singer J, Guyatt GH. Measurement of health status. Ascertaining the minimal clinically important difference. Control Clin Trials. 1989;10(4):407-15.

42. Bhadhuri A, Jowett S, Jolly K, Al-Janabi H. A comparison of the validity and responsiveness of the EQ-5D-5L and SF-6D for measuring health spillovers: a study of the family impact of meningitis. Med Decis Making. 2017;37(8):882-93.

43. Harrison MJ, Davies LM, Bansback NJ, McCoy MJ, Verstappen SMM, Watson K, Symmons DPM, The British Society for Rheumatology Biologics Register Control Centre Consortium, on behalf of the BSRBR. The comparative responsiveness of the EQ-5D and SF-6D to change in patients with inflammatory arthritis. Qual Life Res. 2009:18(9):1195-205.

44. Nolan CM, Longworth L, Lord J, Canavan JL, Jones SE, Kon SS, Man WD. The EQ-5D-5L health status questionnaire in COPD: validity, responsiveness and minimum important difference. Thorax. 2016;71(6):493-500.

45. Björkman L, Musial F, Alraek T, Werner EL, Weidenhammer W, Hamre HJ. Removal of dental amalgam restorations in patients with health complaints attributed to amalgam: a prospective cohort study. J Oral Rehabil. 2020:47(11):1422-34.

46. Stavem K, Augestad LA, Kristiansen IS, Rand K. General population norms for the EQ-5D-3L in Norway: comparison of postal and web surveys. Health Qual Life Outcomes. 2018;16(1):204.
47. Dolan P. Modeling valuations for EuroQol health states. Med Care. 1997;35(11):1095-108.

48. Devlin NJ, Shah KK, Feng Y, Mulhern B, van Hout B. Valuing healthrelated quality of life: an EQ-5D-5L value set for England. Health Econ. 2018:27(1):7-22.

49. Brazier JE, Roberts J. The estimation of a preference-based measure of health from the SF-12. Med Care. 2004:42(9):851-9.

50. Loge HJ, Kaasa S. Short Form 36 (SF-36) health survey: normative data from the general Norwegian population. Scand J Soc Med. 1998;26(4):250-8.

51. Jacobsen EL, Bye A, Aass N, Fosså SD, Grotmol KS, Kaasa S, Loge JH, Moum T, Hjermstad MJ. Norwegian reference values for the Short-Form Health Survey 36: development over time. Qual Life Res. 2018;27(5):1201-12.

52. Sjursen TT, Lygre GB, Dalen K, Helland V, LæGreid T, Svahn J, Lundekvam $B F$, Björkman L. Changes in health complaints after removal of amalgam fillings. J Oral Rehabil. 2011;38(11):835-48.

53. Kaplan MS, Berthelot JM, Feeny D, McFarland BH, Khan S, Orpana H. The predictive validity of health-related quality of life measures: mortality in a longitudinal population-based study. Qual Life Res. 2007;16(9):1539-46.

54. Cronbach LJ, MeehI PE. Construct validity in psychological tests. Psychol Bull. 1955;52(4):281-302.

55. Kazis LE, Anderson JJ, Meenan RF. Effect sizes for interpreting changes in health status. Med Care. 1989;27(3 Suppl):S178-189.

56. Cohen J. Statistical power analysis for the behavioral sciences. Hillsdale, NJ: Lawrence Erlbaum Associates; 1988.

57. Beaton DE, Hogg-Johnson S, Bombardier C. Evaluating changes in health status: reliability and responsiveness of five generic health status measures in workers with musculoskeletal disorders. J Clin Epidemiol. 1997:50(1):79-93.

58. Crosby RD, Kolotkin RL, Williams GR. Defining clinically meaningful change in health-related quality of life. J Clin Epidemiol. 2003;56(5):395-407.

59. de Vet $\mathrm{HC}$, Terwee $\mathrm{CB}$. The minimal detectable change should not replace the minimal important difference. J Clin Epidemiol. 2010;63(7):804-5.

60. de Vet HC, Terwee CB, Ostelo RW, Beckerman H, Knol DL, Bouter LM. Minimal changes in health status questionnaires: distinction between minimally detectable change and minimally important change. Health Qual Life Outcomes. 2006:4:54-54.

61. Norman GR, Sloan JA, Wyrwich KW. Interpretation of changes in healthrelated quality of life: the remarkable universality of half a standard deviation. Med Care. 2003;41(5):582-92.

62. Le QA, Doctor JN, Zoellner LA, Feeny NC. Minimal clinically important differences for the EQ-5D and QWB-SA in Post-traumatic Stress Disorder (PTSD): results from a Doubly Randomized Preference Trial (DRPT). Health Qual Life Outcomes. 2013;11:59.

63. Terluin B, Eekhout I, Terwee CB, de Vet HCW. Minimal important change (MIC) based on a predictive modeling approach was more precise than MIC based on ROC analysis. J Clin Epidemiol. 2015;68(12):1388-96.

64. Mehta CR, Patel NR. Exact logistic regression: theory and examples. Stat Med. 1995;14(19):2143-60.

65. Revicki D, Hays RD, Cella D, Sloan J. Recommended methods for determining responsiveness and minimally important differences for patientreported outcomes. J Clin Epidemiol. 2008:61 (2):102-9.

66. Golicki D, Niewada M, Karlinska A, Buczek J, Kobayashi A, Janssen MF, Pickard AS. Comparing responsiveness of the EQ-5D-5L, EQ-5D-3L and EQ VAS in stroke patients. Qual Life Res. 2015;24(6):1555-63.

67. Barnett AG, van der Pols JC, Dobson AJ. Regression to the mean: what it is and how to deal with it. Int J Epidemiol. 2005;34(1):215-20.

68. Hamre HJ, Glockmann A, Kienle GS, Kiene H. Combined bias suppression in single-arm therapy studies. J Eval Clin Pract. 2008;14(5):923-9.

69. Lamu AN, Gamst-Klaussen T, Olsen JA. Preference weighting of health state values: what difference does it make, and why? Value in Health. 2017:20(3):451-7.

70. Grieve R, Grishchenko M, Cairns J. SF-6D versus EQ-5D: reasons for differences in utility scores and impact on reported cost-utility. Eur J Health Econ. 2009;10(1):15-23. 
71. Pickard AS, Wang Z, Walton SM, Lee TA. Are decisions using cost-utility analyses robust to choice of SF-36/SF-12 preference-based algorithm? Health Qual Life Outcomes. 2005;3:11.

72. van Stel HF, Buskens E. Comparison of the SF-6D and the EQ-5D in patients with coronary heart disease. Health Qual Life Outcomes. 2006;4(1):20.

73. Cunillera O, Tresserras R, Rajmil L, Vilagut G, Brugulat P, Herdman M, Mompart A, Medina A, Pardo Y, Alonso J, et al. Discriminative capacity of the EQ-5D, SF-6D, and SF-12 as measures of health status in population health survey. Qual Life Res. 2010;19(6):853-64.
74. Copay AG, Subach BR, Glassman SD, Polly DW Jr, Schuler TC. Understanding the minimum clinically important difference: a review of concepts and methods. Spine J. 2007:7(5):541-6.

\section{Publisher's Note}

Springer Nature remains neutral with regard to jurisdictional claims in published maps and institutional affiliations.
Ready to submit your research? Choose BMC and benefit from:

- fast, convenient online submission

- thorough peer review by experienced researchers in your field

- rapid publication on acceptance

- support for research data, including large and complex data types

- gold Open Access which fosters wider collaboration and increased citations

- maximum visibility for your research: over $100 \mathrm{M}$ website views per year

At BMC, research is always in progress.

Learn more biomedcentral.com/submissions 